\title{
A nomogram to Predict the Upgrading Rate of ISUP grades of Radical Prostatectomy in Patients Undergoing Transrectal Prostate Biopsy and Transperineal Prostate Cognitive Fusion Biopsy
}

\section{Zhilei Zhang}

Affiliated Hospital of Medical College Qingdao University: The Affiliated Hospital of Qingdao University

\section{Fei Qin}

Affiliated Hospital of Medical College Qingdao University: The Affiliated Hospital of Qingdao University Guofeng Ma

Affiliated Hospital of Medical College Qingdao University: The Affiliated Hospital of Qingdao University Hang Yuan

The Affiliated Hospital of Qingdao University

\section{Yongbo Yu}

The Affiliated Hospital of Qingdao University

\section{Yefeng Sun}

Affiliated Hospital of Medical College Qingdao University: The Affiliated Hospital of Qingdao University

Yanxia Jiang

The Affiliated Hospital of Qingdao University

Wei Jiao

The Affiliated Hospital of Qingdao University

\section{Mingxin Zhang}

The Affiliated Hospital of Qingdao University

Haitao Niu ( $\nabla$ niuhtqyfy123@126.com )

Affiliated Hospital of Medical College Qingdao University

\section{Research}

Keywords: prostate cancer, ISUP upgrade, nomogram

Posted Date: December 15th, 2020

DOl: https://doi.org/10.21203/rs.3.rs-127805/v1

License: (c) (i) This work is licensed under a Creative Commons Attribution 4.0 International License. 
Page $2 / 23$ 


\section{Abstract}

Backgroud: This study was aimed to develop and internally validate an ISUP (International Society of Urology Pathology) upgrade risk nomogram from the biopsy tissue to the specimen of radical prostatectomy.

Methods: The clinical characteristics of 166 patients with prostate cancer were retrospectively analyzed, who were divided into two groups based on the upgrade of ISUP between the biopsy tissue and radical prostatectomy specimen. Logistic regression analysis was used to predict the significant independent factors of ISUP upgrade, a nomogram was established to predict ISUP upgrade of prostatectomy specimen based on the significant factors. The C-index, calibration plot, and decision curve analysis were used to assess the discrimination, calibration, and clinical usefulness of the predicting model. Internal validation was evaluated by using the bootstrapping validation.

Results: There were 47 patients in the ISUP upgrade group and 119 patients in the no ISUP upgrade group respectively. Patients in the ISUP upgrade group inclined to be younger age, smaller PV, lower GS scores and PB-ISUP than the no ISUP upgrade group $(p=0.043, p=0.041, p<0.001, p=0.04$, respectively), Multivariate logistic regression analysis showed that $P S A \geq 20 \mathrm{ng} / \mathrm{ml}(\mathrm{OR}=8.558, P=0.024)$, Gleason score of $\mathrm{PCa} \leq 6$ (OR=9.026, $\mathrm{P}=0.004)$, $\mathrm{PB}-\mathrm{ISUP}=3,4 \mathrm{vs5}$ ( $\mathrm{OR}=23.232, \mathrm{P}=0.000417 ; \mathrm{OR}=26.72, \mathrm{P}=0.000241)$, ways of prostate biopsy (TP-SB+COG-TB (transperineal prostate biopsy + cognitive fusion targeted biopsy) VS TR-SB (transrectal prostate system biopsy), OR=033, $P=0.036$ ) and number of positive cores $<10$ $(\mathrm{OR}=0.21, \mathrm{P}=0.002)$ were the independent risk factors for ISUP upgrade. A prediction nomogram model of ISUP upgrade was built based on these significant factors above, the area under the receiver operating characteristic (AUC) curve of which was 0.843 . The C-index for the prediction nomogram was 0.871 (95\% Cl: 0.817-0.925) and the nomogram showed good calibration. Decision curve analysis also demonstrated that the threshold value of RP-ISUP upgrade risk was $1 \%$ to $89 \%$.

Conclusion: A novel nomogram incorporating PSA, Gleason score of PCa, PB-ISUP, ways of prostate biopsy and number of positive cores was built with a relatively good accuracy to assist clinicians to evaluate the risk of ISUP upgrade in the radical prostatectomy specimen, especially for the low-risk prostate cancer diagnosed by TR-SB.

\section{Introduction}

Prostate cancer ( $\mathrm{PCa}$ ) is a common malignant cancer in middle-aged and elderly men, with the aging process and the popularity of early screening for prostate cancer at home and abroad, the incidence of prostate cancer is on the rise at present ${ }^{[1]}$. The diagnosis of prostate cancer still depends on histopathology obtained by prostate biopsy, which is the gold standard for the diagnosis of prostate cancer. Gleason score of prostate biopsy tissue is considered as an important factor for assessing the prognosis of prostate cancer, which could guide the treatment options for prostate cancer. However, more and more studies have shown that the gleason scores of prostate biopsy tissue and pathological 
specimens after radical prostatectomy varied differently, the coincidence rate of them was only $28 \%-58 \%$, $27 \% \sim 60 \%$ for low gleason score, and $8 \% \sim 32 \%$ for high gleason score ${ }^{[2]}$. In 2014 , the improved gleason score system put forward by the International Society of Urology Pathology (ISUP) was the latest and most accurate grade system for prostate cancer ${ }^{[3,4]}$, which was widely used to assess the malignant degree of prostate cancer, at the same time, the new ISUP grade system evaluated better to guide the next treatment and assess the prognosis of patients with prostate cancer than the previous gleason scores system. However, even based on the 2014 ISUP grade system, the pathology grade of RP specimens was still higher than that of prostate biopsy tissues with an incidence of $20 \% \sim 30 \%[5,6]$.

At present, few studies focus on the ISUP upgrade risk from prostate biopsy tissue to radical prostatectomy specimen, so we analyzed the risk factors of ISUP upgrade after RP compared with biopsy tissue, and a nomogram was established based on the significant risk factors, which could assist clinicians with an important reference when drawing up treatment plans for prostate cancer patients based on the clinical results of prostate biopsy.

\section{Patients And Methods}

\section{Patients}

This study retrospectively analyzed 736 patients with suspected prostate cancer admitted to the Affiliated Hospital of Qingdao University from May 2019 to May 2020, who underwent transrectal or transperineal prostate biopsy to diagnose prostate cancer, and 353 patients were identified to be with prostate cancer.

Exclusion criteria: (1). Patients without radical prostatectomy after prostate biopsy (54 cases); (2). Patients with ADT therapy and other treatments (35 cases); (3). Patients with chronic or acute inflammatory reaction (20 cases); (4). Patients combined with other malignant tumors (17 cases); (5). Patients with incomplete data (61 cases). This study included 166 patients at last, whose data were complete and detailed, the changes of ISUP grade in biopsy and radical prostatectomy were compared so as to identify the predictive factors of ISUP upgrade. This study was approved by the ethics committee of the Affiliated Hospital of Qingdao University.

\section{Methods}

The WHO/ISUP 2014 classification system were divided into five groups, which were group 1: Gleason score $3+3=6 / 10$; Group 2: Gleason score $3+4=7 / 10$; Group 3: Gleason score $4+3=7 / 10$; Group 4: Gleason total score 8 and Group 5: Gleason total score $9-10^{[3]}$. The ISUP upgrade group was defined as the increase grade of ISUP in RP specimen compared with prostate biopsy tissue. Then the patients were divided into the ISUP upgrade group $(n=47)$ and the ISUP non-upgrade group $(n=119)$ according to the postoperative ISUP grade, and the independent factors for the prediction of ISUP elevation were analyzed. All pathologic diagnosis were identified by at least 2 pathologists. All patients were informed about the 
procedure of prostate biopsy and written informed consent were obtained. They were randomly selected to perform prostate biopsy according to the random number method, which included transrectal ultrasound (TRUS)-guided prostate biopsy (TR-SB) and transperineal cognitive fusion targeted biopsy (TP-SB + COG-TB). Radical prostatectomy was performed in patients diagnosed with prostate cancer after biopsy.

All patients underwent mpMRI on a 1.5-T MRI or 3.0-T MRI, and patients had at least three sequencestriplanar T2 weighted, diffusion weighted imaging (DWI) and apparent diffusion coefficient (ADC), region of interest was identified on MRI by our surgeon during preoperative preparation.

\section{Biopsy}

Transrectal prostate system biopsy (TR-SB): Systematic biopsy include 12-15 slices was obtained from apex, apex lateral, mid, mid lateral, base and base lateral of right and left prostatic lobes of prostate $\mathrm{e}^{[7]}$, the biopsy number might vary according to the prostate volume or additional suspicious transrectal ultrasound findings. Biopsy tissue were fixed in a separate glass bottle containing $10 \%$ formaldehyde solution

Transperineal prostate biopsy + cognitive fusion targeted biopsy (TP-SB + COG-TB): Systematic biopsy was typically 12 cores collected in the medial and lateral aspects of the apical, mid, and base of the prostate on the left and right side, COG-TB was that 2 to 4 samples were taken from each suspicious lesion ${ }^{[8]}$. Biopsy tissue were also fixed in a separate glass bottle containing $10 \%$ formaldehyde solution.

\section{Data Collection}

Preoperative and postoperative clinical characteristics and histopathological results were evaluated for each patient. Upgrade of ISUP and no ISUP upgrade of the tumor from biopsy to final pathology were recorded for each patient. Patient's records including age, PSA, PV, PSAD, Prostate biopsy, Total biopsy cores, Number of positive cores, PB-ISUP, Biopsy tumor percentage, T stage, Gleason score of PCa and PSM (positive surgical margin) were retrieved from the hospital database for retrospective analysis.

\section{Statistics}

Data was analyzed by IBM SPSS Statistics ver. 22.0 (IBM Corp., Armonk, NY, USA) and R 4.0.2 (formerly AT\&T, now Lucent Technologies). Continuous variables were analyzed by using Student $t$ test in variables with normal distribution. Categorical variables were compared using Chi-square test and Mann-Whitney $U$ test. Uni and multiply logistic regression model for analyzing the effects of patients' clinical characteristics between ISUP Upgrade and No ISUP upgrade. The independent factors for the prediction of pathological escalation were identified and a nomogram model was established. The prediction of nomogram was evaluated by ROC curve (receive operating characteristic) and AUC (area under the 
curve). Bootstrap resampling was used to verify the model, at the same time, the calibration curve and DCA (decision curve analysis) were draw to evaluate the model. All $P$ values were two-sided, and a difference of $\mathrm{P}<0.05$ was considered statistically significant.

\section{Results}

166 patients were evaluated in our study, comprising 47 patients in the ISUP upgrade group and 119 patients in the no ISUP upgrade group based on the change of ISUP grade. The mean age of patients was $68.99 \pm 7.61$, and the mean PSA of patients was $37.93 \pm 50.77$. The patient's clinical and histopathologic characteristics were demonstrated in Table 1. As shown in the table, patients in the ISUP upgrade group showed younger age, smaller PV, lower GS scores and PB-ISUP than the no ISUP upgrade group ( $p=$ $0.043, p=0.041, p<0.001, p=0.04$, respectively), in addition, more patients in the TR-SB group inclined to the ISUP upgrade group than the TP-SB + COG-TB group $(P=0.007)$.

There were 66 patients and 100 patients underwent TP-SB + COG-TB and TR-SB, respectively. Table 2 showed the Gleason score of PCa patients in the two ways of prostate biopsy groups. It demonstrated that TP-SB + COG-TB found more csPCa of patients (GS $\geq 7$ ) than the group of TR-SB ( $44.8 \% \mathrm{VS} 38.8 \%, P$ $=0.112$ ), but there were no significant differences in the detection rate of csPCa and PCa between the two prostate biopsy ways.

Table 3 and Fig. 1 showed the differences between the pathology ISUP of preoperative biopsy and pathological specimen, 47 of 166 patients were found with the ISUP group upgrade, whereas no ISUP upgrade was observed in the remaining 119 patients. The distribution of ISUP group of pathological specimen were 13 patients in Group 1, 39 patients in Group 2, 25 patients in Group 3, 24 patients in Group 4,65 patients in Group 5, respectively. The concordance from PB-ISUP group to RP-ISUP group was highest for GR5 (89.36\%) and lowest for GR1 (39.4\%), and 34.78\% of patients in the biopsy GR 2, 38.46\% patients in GR3 and $32.43 \%$ patients in GR 4 were upgraded respectively.

The results of the univariate and multivariate logistic regression analyse were displayed on the Table 4. A younger age, a higher preoperative PSA level, a smaller PV, a higher PSAD, ways of prostate biopsy, a fewer number of positive biopsy cores as well as percentage of cores, lower PB-ISUP grade and PB-GS predicted ISUP upgrade in the univariable analysis. In the multivariable analysis, $P S A \geq 20(O R=8.558, P$ $=0.024)$, PB-gleason score $\leq 6(O R=9.026, P=0.004)$, PB-ISUP $₫ 5(O R=26.72, P \unrhd 0.001)$, ways of prostate biopsy $(O R=0.33, P=0.036)$ and number of positive cores $₫ 10(O R=0.21, P=0.002)$ were found as the independent predictors to the RP-ISUP upgrade.

Based on the results of independent prognostic factors obtained by multivariate logistic regression analysis, a predicted model that incorporated the above independent predictors was constructed to 
predict the probability of postoperative ISUP upgrade of the specimen, which was developed and presented as the nomogram (Fig. 2).

The calibration curve of this RP-ISUP upgrade risk nomogram for the prediction of ISUP upgrade in prostate biopsy patients could well demonstrate the risk of ISUP upgrade after radical prostatectomy (Fig. 3), which displayed a high consistency between predicted and measured values. The C-index for the prediction nomogram was 0.871 (95\% Cl: 0.817-0.925) for our patients, moreover, the prediction nomogram was evaluated by ROC curve (Fig. 4), and the AUC (area under curve) of the ROC was 0.843 . The predicted value of the nomogram was in further certified to be 0.831 through bootstrapping validation.

Figure 5 showed the decision curve of the predicted nomogram, and a positive net benefit was obtained in the range of threshold probabilities ranging from 0.01 to 0.89 in the model, using this RP-ISUP upgrade nomogram in the current study to predict RP-ISUP upgrade risk adds more benefit than the interventionall-patients scheme or the intervention-none scheme.

\section{Discussion}

Prostate cancer is the second most common cancer in males in the worldwide, which leads a high death rate of male ${ }^{[9,10]}$. Although PSA has been used as an indicator to screen for prostate cancer ${ }^{[11]}$, there were still some patients missed out. Moreover, even if patients were diagnosed to be prostate cancer by prostate biopsy, some patients were usually underestimated or overestimated for some subjective and objective reasons, which could result in delayed treatment or overtreatment. Biopsy sample could not reflect the overall pathological characteristics of the disease, and previous studies have reported the inconsistency in gleason score between prostate biopsy tissues and radical specimens ${ }^{[12]}$. It was reported that about $30 \%$ of patients with low-risk prostate cancer had aggressive factors in their specimens after radical surgery ${ }^{[13]}$. In order to identify the risk stratification precisely for prostate cancer, the International Society of Urological Pathology has proposed the latest version of prostate cancer grouping system as an update of the traditional 2005 gleason grading system in November 2014[14], which was accepted by WHO in 2016. Studies have confirmed that the 2014 ISUP group system could more accurately predict the cancer-specific survival time in prostate cancer and the rate of biochemical recurrence after radical prostatectomy ${ }^{[14,15]}$. In addition, The 2014 ISUP grouping system could be able to reduce the incidence of postoperative pathological upgrading, although $19.5 \%$ of patients still had clinically significant grade upgrade ${ }^{[16]}$. The group of ISUP upgrade in our study included 47 patients, which was equal to $28.3 \%$ of the total patients. Brassetti found that $41.4 \%$ of patients from europeans had the post-pathological grade upgrade ${ }^{[17]}$, which was higher than our study, this may be due to the multifocal growth of prostate cancer with high heterogeneity ${ }^{[18]}$. At the same time, different prostate biopsy methods will also appear the discrepancy of pathological grade between biopsy and radical prostatectomy. 
In recent years, some predictive models and clinical parameters included PSA, biopsy and clinical staging have been used to assess the postoperative pathological escalation risk of prostate cancer ${ }^{[19-22]}$, but there were no widely accepted models. The ability of various models to predict the upgrade of pathology remained limited at present, and previous models rarely incorporated the factor of different ways of prostate biopsies, similarly we analyzed the clinical and pathological parameters to affect the ISUP group upgrade in patients with prostate cancer in our study. We found that PSA, gleason score of PCa, PB-ISUP, ways of prostate biopsy and number of positive cores were the significant factors to influence the ISUP group upgrade in the postoperative pathology. Based on the independent factors above, a nomogram model was built to predict the ISUP group upgrade, and there was a good discrimination and calibration power emerged by internal validation in the cohort, moreover, the high C-index and AUC demonstrated that it could be able to widely be accepted and used in the clinic. Compared with previous research ${ }^{[22]}$, this study included more predictive factors, which ensured that the prediction model established in this study was more accurate and credible.

In general, radical prostatectomy is not suitable for patients with low-risk prostate cancer, these patients usually cured by regular active monitoring at present. However, some students have pointed out that postoperative pathological upgrading would exist in these patients. Therefore, accurate examination results before surgery and precise prediction of postoperative pathological results were of great significance to the decisions of further treatment. It was reported that postoperative pathological upgrade was related to younger age patients with prostate cancer ${ }^{[23]}$. This was similar with our study, although the age in our study was not the independent factor for the ISUP-upgrade, the age in the group of ISUPupgrade was relative younger than the no ISUP upgrade group.

PSA was an important indicator for prostate cancer screening, which was also used to evaluate the clinical stratified risk of prostate cancer. With the relationship between PSA and the risk of classification on prostate cancer, scholars in domestic found that serum PSA level was positively correlated with Gleason score. Many foreign studies also have pointed out that patients with higher serum PSA level were more likely to have higher Gleason score after radical surgery ${ }^{[24,25]}$. This was in line with our study, patients with PSA $\geq 20$ in our study tended to be more aggressive than those with lower PSA, and ISUP group of those patients cline to upgrade in our study. So it was an important factor to guide the next step of clinical treatment.

Besides the PSA, gleason score of PCa, PB-ISUP, ways of prostate biopsy and number of positive cores were associated with greater odds of ISUP upgrade in men undergoing subsequent biopsies as part of AS (active surveillance), and we found that these five factors were all related with the prostate biopsy, results showed that the TP-SB + COG-TB were more accurate in the ISUP of prostate biopsy tissue than the TR$\mathrm{SB}$, systemic prostate biopsy guided by transrectal ultrasound were the most common prostate biopsy to diagnose prostate cancer ${ }^{[26]}$. However, systematic prostate biopsy was less sensitive to discover the prostate cancer with high degree of malignancy, and about the pathological ISUP of $25 \%$ 30\% low-risk patients with prostate cancer after radical prostatectomy upgraded compared with the biopsy pathology, 
especially for patients with anterior tumor and large prostate ${ }^{[27]}$. mpMRI had high sensitivity and specificity to screen prostate cancer, TRUS-MRI fusion prostate biopsy has been widely carried out in many countries, although the non-template guided freehand transperineal cognitive fusion prostate biopsy was not accurate but convenient for doctor to perform ${ }^{[27]}$, and our data showed that TP-SB + COGTB could discover more csPCa than TR-SB in our study(44.8\%VS38.8\%), and the ISUP upgrade usually appeared in the patients with non-clinical significant prostate cancer, target biopsy was performed at the core of every lesion to obtain large proportion of tumor tissue, which could not only improve the detection rate of csPCa, but also reduce the probability of pathology escalation after radical surgery. It could provide clinicians with more accurate information in order to make the most suitable treatment for patients.

Therefore, accurate prognostic assessment would assist doctors to reevaluate the patients with ISUP disparities and take timely interventions to prevent testing in low-risk situations, which could also avoid delays or discontinuity in treatment when there was a high probability of a favorable net benefit. The prediction model was very conducive to the accurate assessment of the disease for the low-risk prostate cancer patients with active monitoring and simple conservative treatment. Moreover, the prediction model of this study included many significant clinical factors, which had strong predictive value through internal verification.

There were also some limitations, first, this was a respective, single center study. Second, long-term monitorization to evaluate cancer-specific survival, biochemical recurrence and metastasis were not performed. In addition, although the overall accuracy of our model was higher than that of previous models, the performance of our model needed to be further verified in the external data set.

\section{Conclusion}

A novel nomogram was built with a relatively good accuracy to assist clinicians to evaluate the risk of ISUP upgrade in the radical prostatectomy specimen, especially for the next treatment of patients with low-risk prostate cancer, and this study showed that the ISUP of tissue obtained by transperineal prostate biopsy could coincide well with that of specimen by radical prostatectomy, but this model needs further research and external data validation were needed to identify the accuracy of detection.

\section{Declarations}

Ethics approval: This study was approved by the Ethic Committee of the Affiliated Hospital of Qingdao University.

Consent to participate: Informed consent was obtained in both written and verbal format from patients or guardian for participants under 16 years old to participate.

Informed consent: Not applicable. 
Availability of data and material: Records and data pertaining to this study are in the patient's secure medical records in the Affiliated Hospital of Qingdao University

Disclosure of potential conflicts of interest: Not applicable.

Funding: National Natural Science Foundation and Taishan Scholar Program of Shandong Province.

\section{Authors' contributions:}

Protocol/project development: HT Niu and MX Zhang

Data collection or management: ZL Zhang, F Qin, GF Ma, H Yuan, YB Yu, YF Sun, YX Jiang and W Jiao Data analysis: ZL Zhang, F Qin, GF Ma, YX Jiang and W Jiao

Manuscript writing/editing Data collection: ZL Zhang

All authors commented on previous versions of the manuscript. All authors read and approved the final manuscript.

Acknowledgement: This work was supported by the National Natural Science Foundations of China under Grant 81772713, 81472411, 81981260351, 81972378; Taishan Scholar Program of Shandong Province under Grant tsqn20161077; Natural Science Foundation of Shandong Province under Grant ZR2016HQ18; Key Research and Development Program of Shandong Province under Grant 2018 GSF118197.

\section{References}

1. Chen W, Zheng R, Baade PD, et al. Cancer statistics in China, 2015. CA Cancer J Clin. 2016. 66(2).

2. Noguchi M, Stamey TA, Mcneal JE, Yemoto CM. Relationship between systematic biopsies and histological features of 222 radical prostatectomy specimens: lack of prediction of tumor significance for men with nonpalpable prostate cancer. J Urol. 2001;166(1):104-10.

3. Epstein JI, Egevad L, Amin MB, Delahunt B, Srigley JR, Humphrey PA. The 2014 International Society of Urological Pathology (ISUP) Consensus Conference on Gleason Grading of Prostatic Carcinoma. Am J Surg Pathol. 2015: 1.

4. Optimization of the 2014. Gleason grade grouping in a Canadian cohort of patients with localized prostate cancer. BJU Int. 2019. 123(4).

5. Athanazio D, Gotto G, Shea-Budgell M, Yilmaz A, Trpkov K. Global Gleason grade groups in prostate cancer: concordance of biopsy and radical prostatectomy grades and predictors of upgrade and downgrade. Histopathology. 2017.

6. The new. Epstein gleason score classification significantly reduces upgrading in prostate cancer patients. European Journal of Surgical Oncology the Journal of the European Society of Surgical 
Oncology \& the British Association of Surgical Oncology. 2018.

7. Guo LH, Wu R, Xu HX, et al. Comparison between Ultrasound Guided Transperineal and Transrectal Prostate Biopsy: A Prospective, Randomized, and Controlled Trial. Sci Rep. 2015;5:16089.

8. Scattoni V, Russo A, Di Trapani E, Capitanio U, La Croce G, Montorsi F. Repeated biopsy in the detection of prostate cancer: when and how many cores. Arch Ital Urol Androl. 2014;86(4):311-3.

9. Zhou CK, Check DP, Lortet-Tieulent J, et al. Prostate cancer incidence in 43 populations worldwide: An analysis of time trends overall and by age group. Int J Cancer. 2016;138(6):1388-400.

10. Ferlay J, Shin HR, Bray F, Forman D, Mathers C, Parkin DM. Estimates of worldwide burden of cancer in 2008: GLOBOCAN 2008. Int J Cancer. 2010;127(12):2893-917.

11. Miller DC, Gruber SB, Hollenbeck BK, Montie JE, Wei JT. Incidence of initial local therapy among men with lower-risk prostate cancer in the United States. J Natl Cancer Inst. 2006;98(16):1134-41.

12. Epstein JI, Feng Z, Trock BJ, Pierorazio PM. Upgrading and downgrading of prostate cancer from biopsy to radical prostatectomy: incidence and predictive factors using the modified Gleason grading system and factoring in tertiary grades. Eur Urol. 2012;61(5):1019-24.

13. Beauval JB, Ploussard G, Soulié $M$, et al. Pathologic findings in radical prostatectomy specimens from patients eligible for active surveillance with highly selective criteria: a multicenter study. Urology. 2012;80(3):656-60.

14. Epstein JI, Amin MB, Reuter VE, Humphrey PA. Contemporary Gleason Grading of Prostatic Carcinoma: An Update With Discussion on Practical Issues to Implement the 2014 International Society of Urological Pathology (ISUP) Consensus Conference on Gleason Grading of Prostatic Carcinoma. Am J Surg Pathol. 2017. 41(4): e1.

15. Pompe RS, Davis-Bondarenko H, Zaffuto E, et al. Population-Based Validation of the 2014 ISUP Gleason Grade Groups in Patients Treated With Radical Prostatectomy, Brachytherapy, External Beam Radiation, or no Local Treatment. Prostate. 2017;77(6):686.

16. Nunzio CD, Pastore AL, Lombardo R, et al. The new Epstein Gleason score classification significantly reduces upgrading in prostate cancer patients. European Journal of Surgical Oncology. 2018: S0748798318300040.

17. Brassetti A, Lombardo, et al. Prostate-specific Antigen Density Is a Good Predictor of Upstaging and Upgrading, According to the New Grading System: The Keys We Are Seeking May Be Already in Our Pocket. UROLOGY -NEW JERSEY THEN NEW YORK-. 2018.

18. Humphrey, Peter A. Complete histologic serial sectioning of a prostate gland with adenocarcinoma. Am J Surg Pathol. 1993;17(5):468-72.

19. Bud? Us L, Graefen M, Salomon G, et al. The novel nomogram of Gleason sum upgrade: Possible application for the eligible criteria of low dose rate brachytherapy. International Journal of Urology Official Journal of the Japanese Urological Association. 2010;17(10):862-8.

20. Wang JY, Zhu Y, Wang CF, Zhang SL, Dai B, Ye DW. A nomogram to predict Gleason sum upgrading of clinically diagnosed localized prostate cancer among Chinese patients. Chin J Cancer. 2014;33(005):241-8. 
21. Chun KH, Steuber T, Erbersdobler A, et al. Development and Internal Validation of a Nomogram Predicting the Probability of Prostate Cancer Gleason Sum Upgrading Between Biopsy and Radical Prostatectomy Pathology. Eur Urol. 2006;49(5):820-6.

22. He B, Chen R, Gao X, Ren S, Sun Y. Nomograms for predicting Gleason upgrading in a contemporary Chinese cohort receiving radical prostatectomy after extended prostate biopsy: development and internal validation. Oncotarget. 2016. 7(13).

23. Turgay T. Berrin, et al. The factors predicting upgrading of prostate cancer by using International Society for Urological Pathology (ISUP) 2014 Gleason grading system. Turk J Urol. 2018.

24. Gershman B, Dahl DM, Olumi AF, Young RH, Mcdougal WS, Wu CL. Smaller prostate gland size and older age predict Gleason score upgrading. Urol Oncol. 2013;31(7):1033-7.

25. Feng Z, Trock BJ, Epstein JI, Pierorazio PM. Upgrading and downgrading of prostate cancer from biopsy to radical prostatectomy: Incidence and predictive factors using the modified gleason grading system and factoring in tertiary grades. Eur Urol. 2012;61(5):1019-24.

26. Heidenreich A, Bastian PJ, Bellmunt J, et al. EAU Guidelines on Prostate Cancer. Part 1: Screening, Diagnosis, and Local Treatment with Curative Intent-Update 2013. Eur Urol. 2014;65(1):124-37.

27. Siddiqui MM, Rais-Bahrami S, Truong $\mathrm{H}$, et al. Magnetic Resonance Imaging/Ultrasound-Fusion Biopsy Significantly Upgrades Prostate Cancer Versus Systematic 12-core Transrectal Ultrasound Biopsy. Eur Urol. 2013;64(5):713-9.

\section{Tables}

Due to technical limitations, table 1 to 4 is only available as a download in the Supplemental Files section.

\section{Figures}




\section{Grade group}

$120.00 \%$

$100.00 \%$

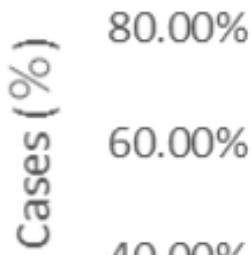

$40.00 \%$

$20.00 \%$

$0.00 \%$
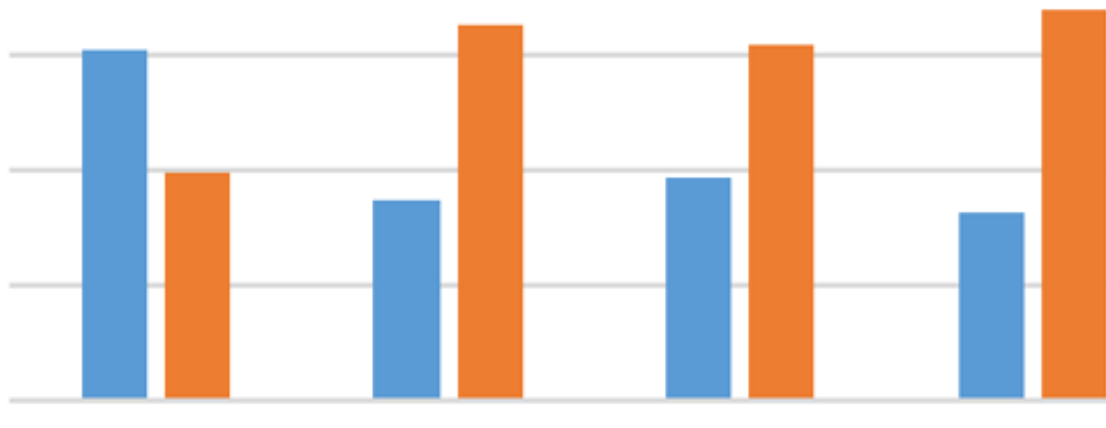

GR1

GR2

GR3

GR4

GR5

- Upgrade $\quad$ No upgrade

Figure 1

Concordance for individual ISUP grade groups 1-5 from biopsy to radical prostatectomy. 


\section{Grade group}

$120.00 \%$

$100.00 \%$

$\frac{2}{0}$

$80.00 \%$

$60.00 \%$

$40.00 \%$

$20.00 \%$

$0.00 \%$
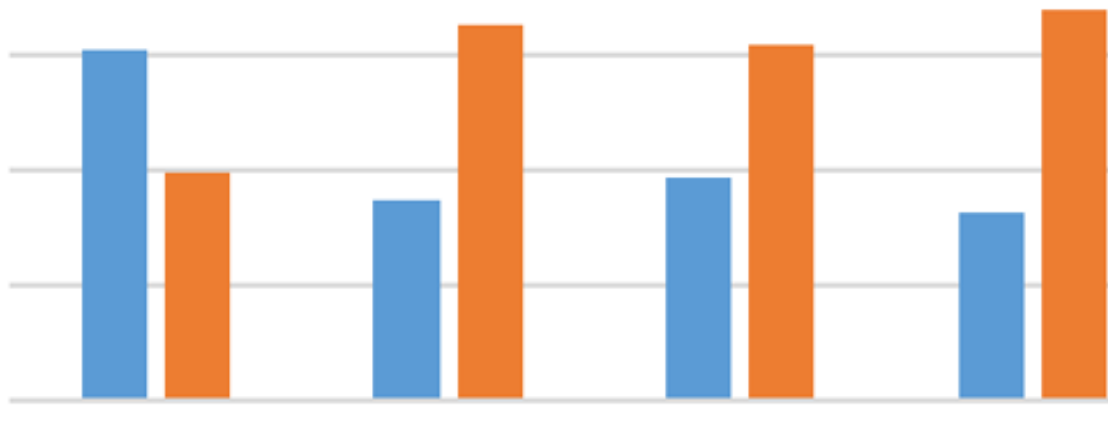

GR1

GR2

GR3

GR4

GR5

- Upgrade $\quad$ No upgrade

Figure 1

Concordance for individual ISUP grade groups 1-5 from biopsy to radical prostatectomy. 
Points

$\begin{array}{llllllllll}0 & 10 & 20 & 30 & 40 & 50 & 60 & 70 & 80 & 90\end{array}$

PSA

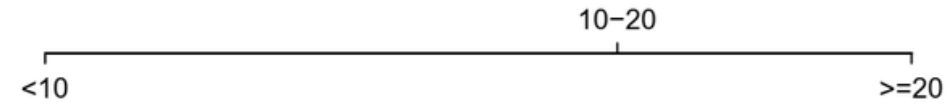

Prostate_biopsy

TR

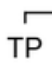

Total_core_percentage

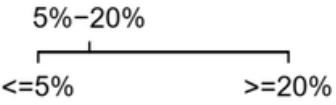

Number_of_positive_cores $>10 \quad>=10$

Gleason_score_of_PCa $>>=6$

PB_ISUP_group

1

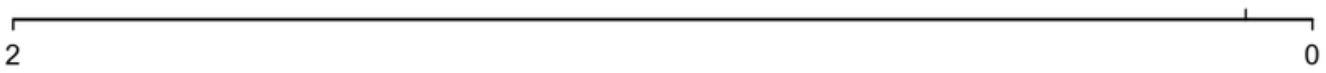

Total Points

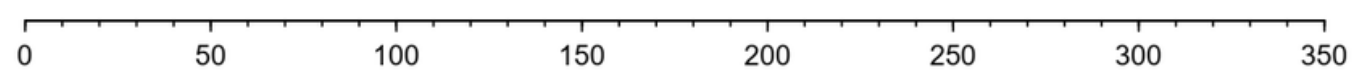

Risk of upgrade

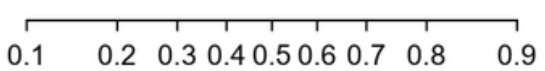

Figure 2

RP-ISUP upgrade nomogram Note: The RP-ISUP upgrade nomogram was developed in the cohort, with the use of PSA, Prostate biopsy, Total core percentage, Number of positive cores, Gleason score of PCa and PB-ISUP group. Abbreviations: PSA: prostate antigen; GS: gleason score; PB: prostate biopsy; ISUP: International Society of Urologic Pathology; 
Points

0 10 20 30 40 50 60

70

80 $90 \quad 100$ PSA

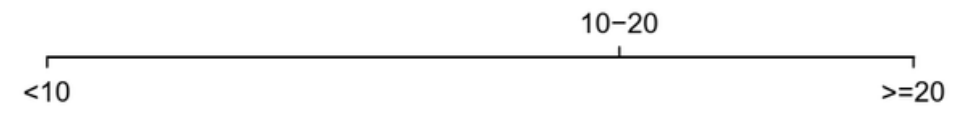

Prostate_biopsy

TR

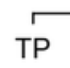

Total_core_percentage

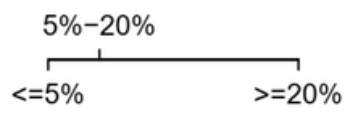

Number_of_positive_cores $>10 \quad>=10$

Gleason_score_of_PCa $>>=6$

PB_ISUP_group

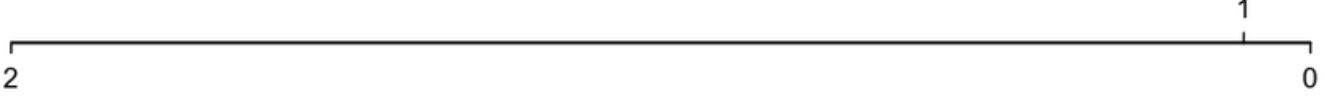

Total Points

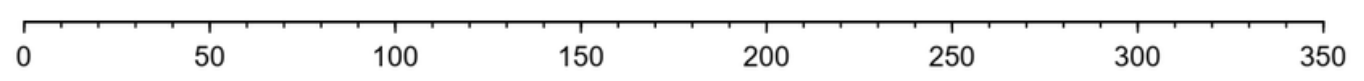

Risk of upgrade

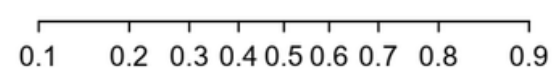

Figure 2

RP-ISUP upgrade nomogram Note: The RP-ISUP upgrade nomogram was developed in the cohort, with the use of PSA, Prostate biopsy, Total core percentage, Number of positive cores, Gleason score of PCa and PB-ISUP group. Abbreviations: PSA: prostate antigen; GS: gleason score; PB: prostate biopsy; ISUP: International Society of Urologic Pathology; 


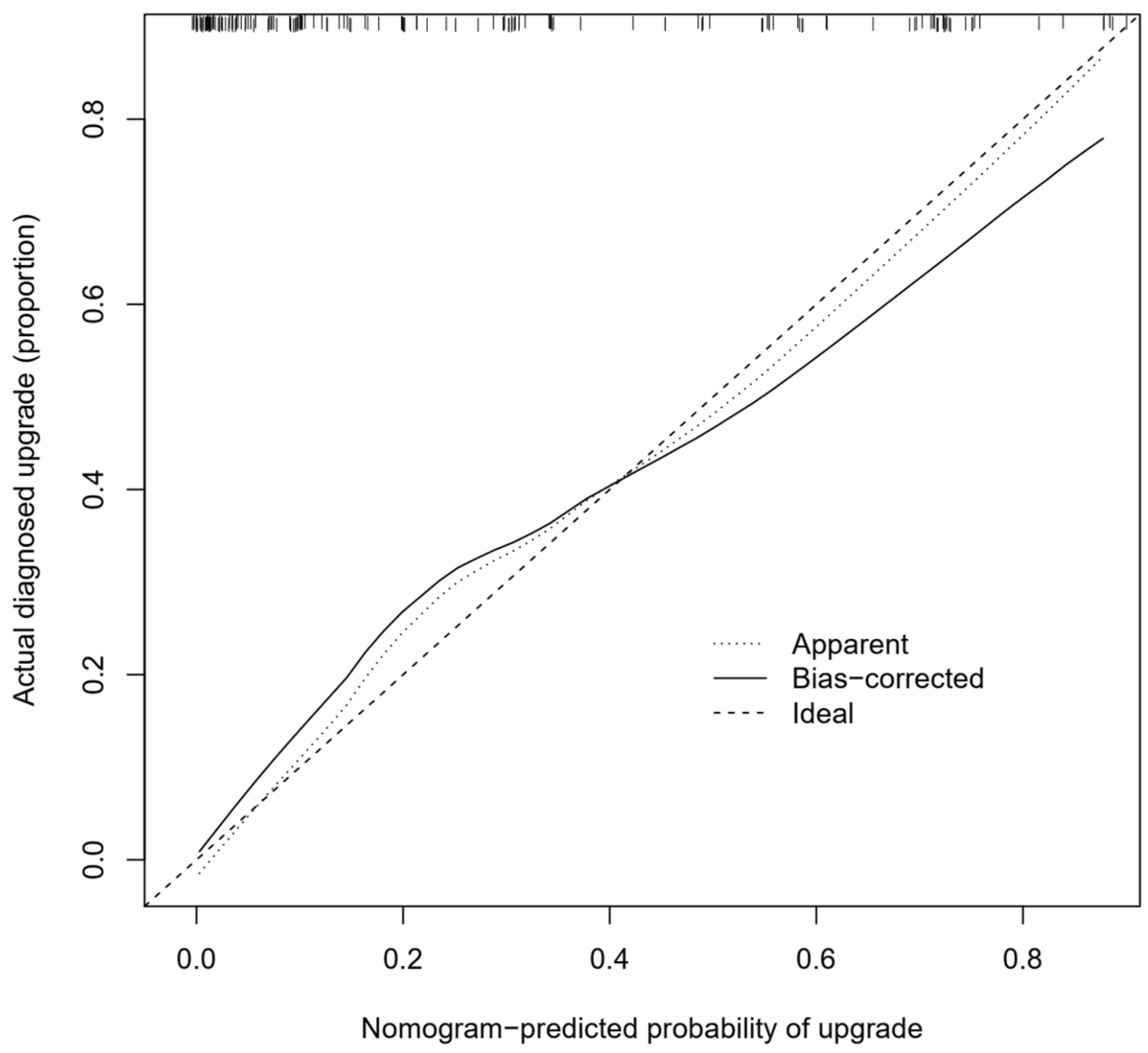

Figure 3

Calibration curves of the RP-ISUP upgrade nomogram prediction in the cohort. Notes: The x-axis represents the predicted RP-ISUP upgrade risk. The y-axis represents the actual diagnosed RP-ISUP upgrade proportion. The diagonal dotted line represents a perfect prediction by an ideal model. The solid line represents the performance of the nomogram, of which a closer fit to the diagonal dotted line represents a better prediction. 


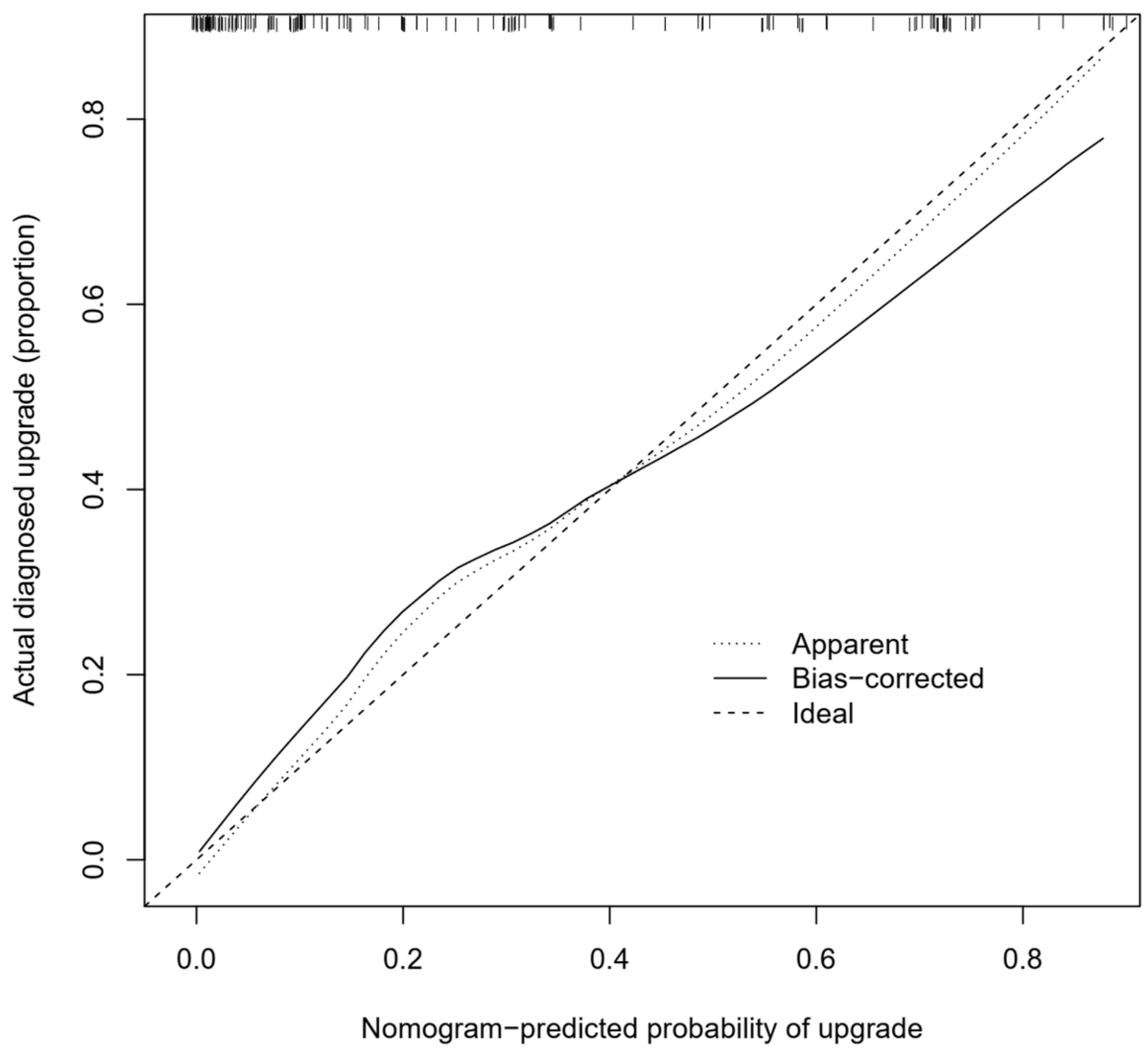

Figure 3

Calibration curves of the RP-ISUP upgrade nomogram prediction in the cohort. Notes: The x-axis represents the predicted RP-ISUP upgrade risk. The y-axis represents the actual diagnosed RP-ISUP upgrade proportion. The diagonal dotted line represents a perfect prediction by an ideal model. The solid line represents the performance of the nomogram, of which a closer fit to the diagonal dotted line represents a better prediction. 
AUS $=\mathbf{0 . 8 4 3}$

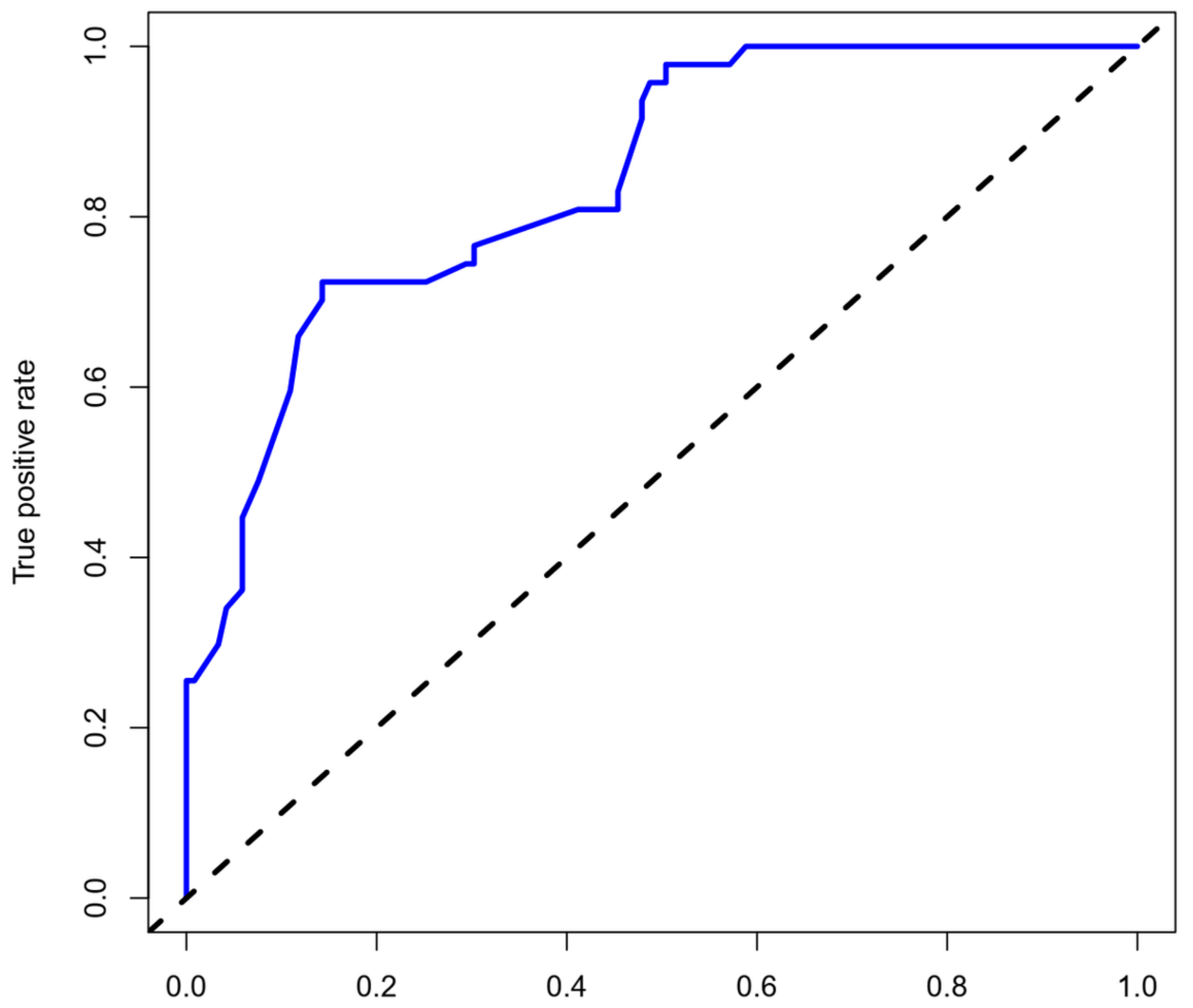

False positive rate

Figure 4

The ROC curve of the Nomogram model to predict the RP-ISUP upgrade.

Page 19/23 
AUS $=\mathbf{0 . 8 4 3}$

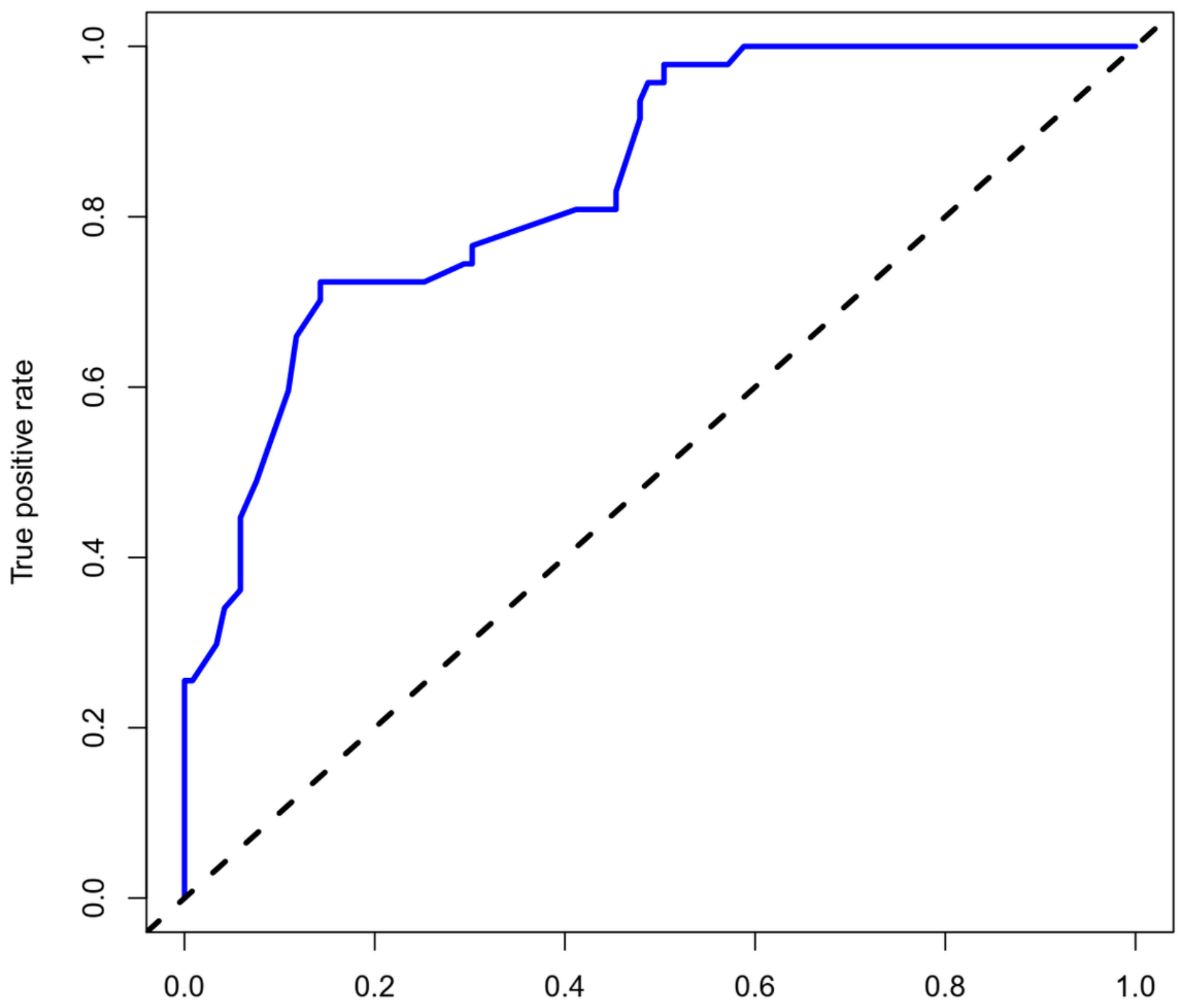

False positive rate

Figure 4

The ROC curve of the Nomogram model to predict the RP-ISUP upgrade.

Page 20/23 


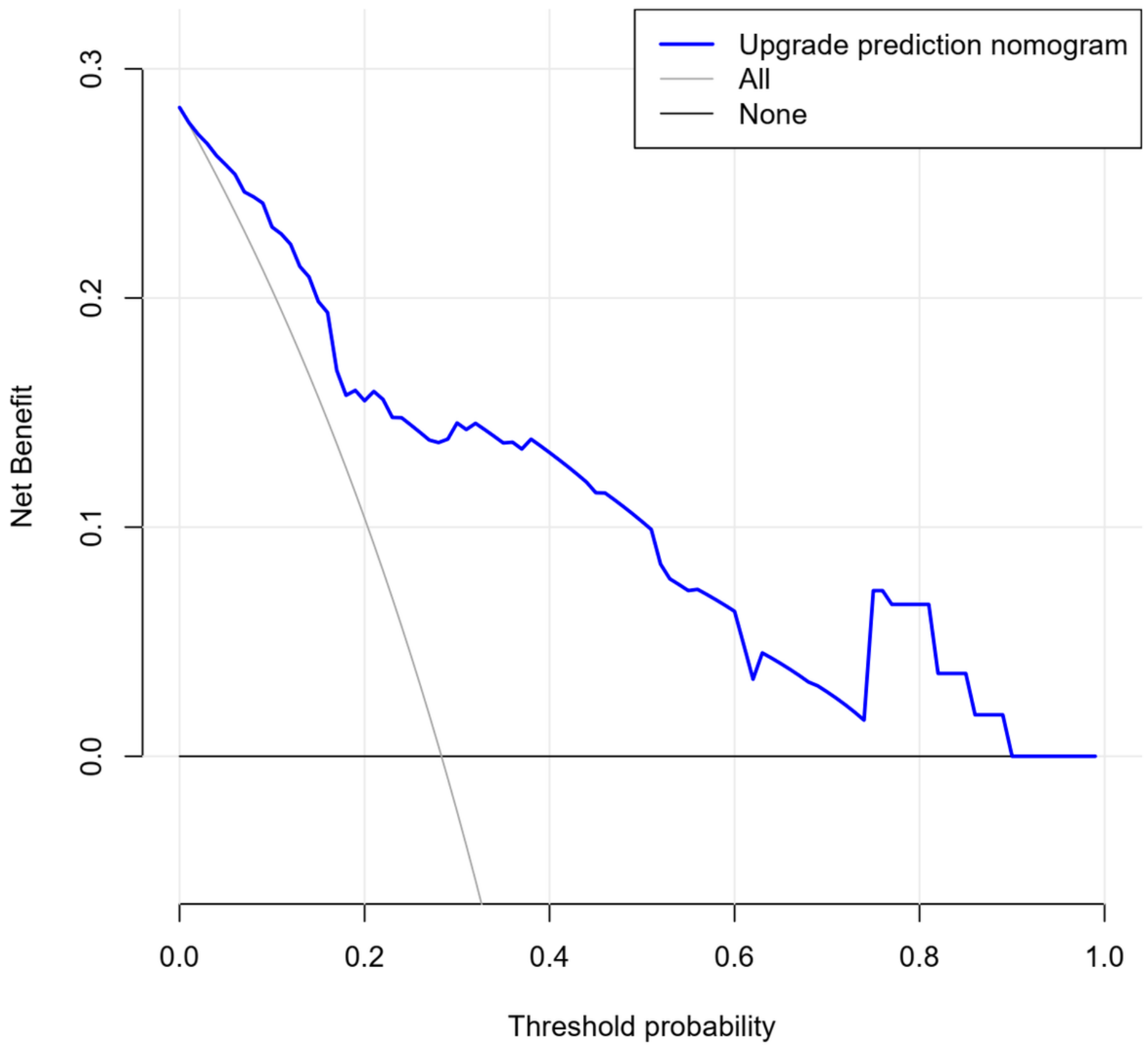

\section{Figure 5}

Decision curve analysis for the RP-ISUP upgrade nomogram. Notes: The y-axis measures the net benefit. The dotted line represents the RP-ISUP upgrade risk nomogram. The thin solid line represents the assumption that all patients are RP-ISUP upgrades. Thin thick solid line represents the assumption that no patients are no upgrade to RP-ISUP. The decision curve showed that the threshold value of RP-ISUP upgrade risk is $1 \%$ to $89 \%$, using this RP-ISUP upgrade nomogram in the current study to predict RP-ISUP upgrade risk adds more benefit than the intervention-all-patients scheme or the intervention-none scheme. 


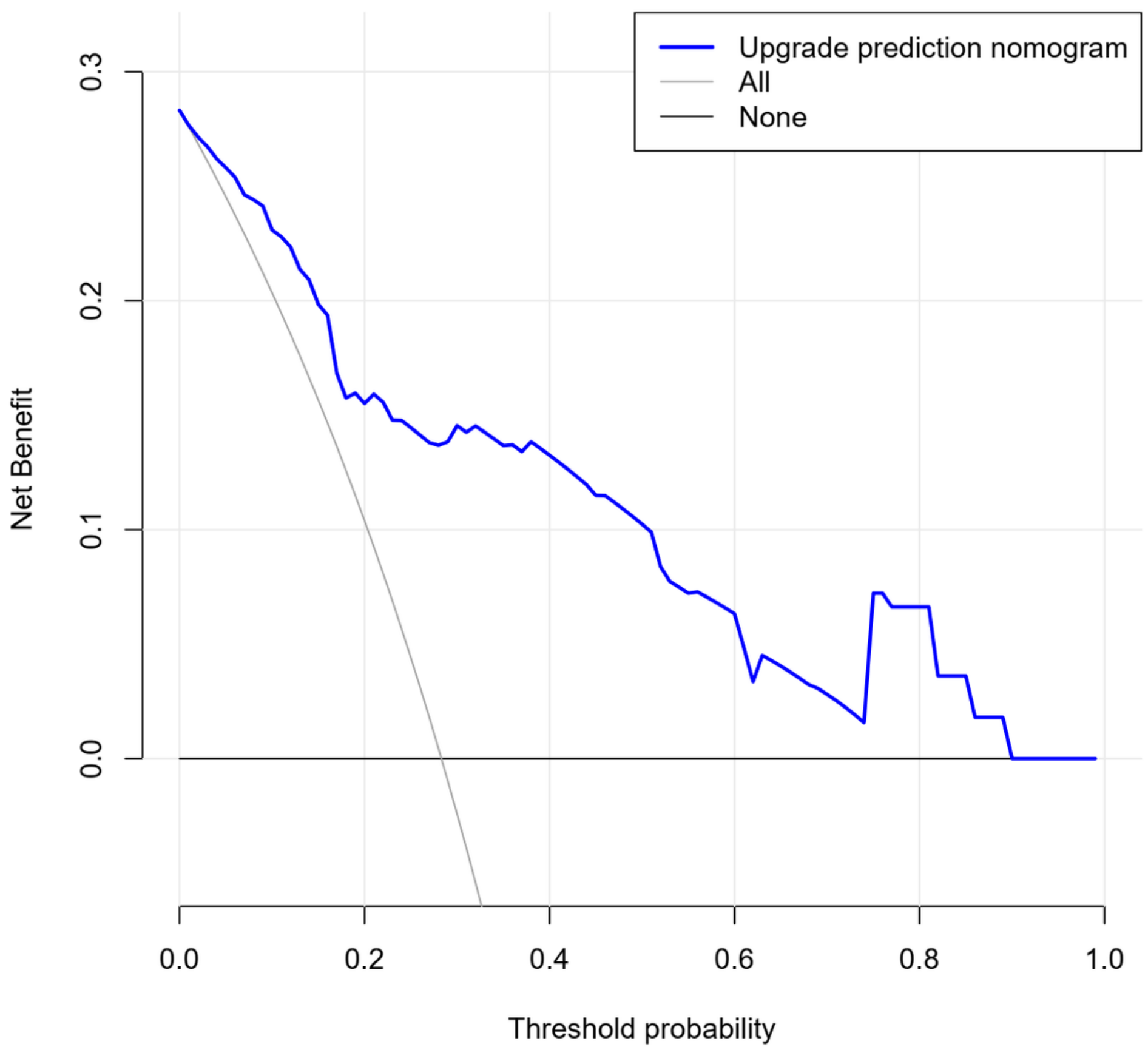

\section{Figure 5}

Decision curve analysis for the RP-ISUP upgrade nomogram. Notes: The y-axis measures the net benefit. The dotted line represents the RP-ISUP upgrade risk nomogram. The thin solid line represents the assumption that all patients are RP-ISUP upgrades. Thin thick solid line represents the assumption that no patients are no upgrade to RP-ISUP. The decision curve showed that the threshold value of RP-ISUP upgrade risk is $1 \%$ to $89 \%$, using this RP-ISUP upgrade nomogram in the current study to predict RP-ISUP upgrade risk adds more benefit than the intervention-all-patients scheme or the intervention-none scheme. 


\section{Supplementary Files}

This is a list of supplementary files associated with this preprint. Click to download.

- Tables.docx

- Tables.docx 